\title{
Rapid In Situ Polymerization of Polyacrylonitrile/Graphene Oxide Nanocomposites as Precursors for High- Strength Carbon Nanofibers
}

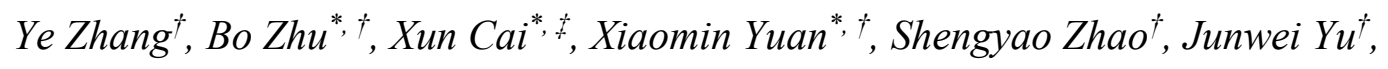
Kun Qiao ${ }^{\Uparrow}$ Rongman Qin ${ }^{\dagger}$

${ }^{\dagger}$ Key Laboratory for Liquid-Solid Structural Evolution and Processing of Materials

(Ministry of Education), School of Materials Science and Engineering, Shandong University, Jinan 250061, China. ${ }^{\star}$ School of Computer Science and Technology, Shandong University, Jinan 250101, China. \School of Mechanical, Electrical \& Information Engineering, Shandong University, Weihai 264209, China.

*E-mail: zhubo@sdu.edu.cn (B. Zhu); caixunzh@sdu.edu.cn (X. Cai); 124143739@qq.com (X. M. Yuan) 


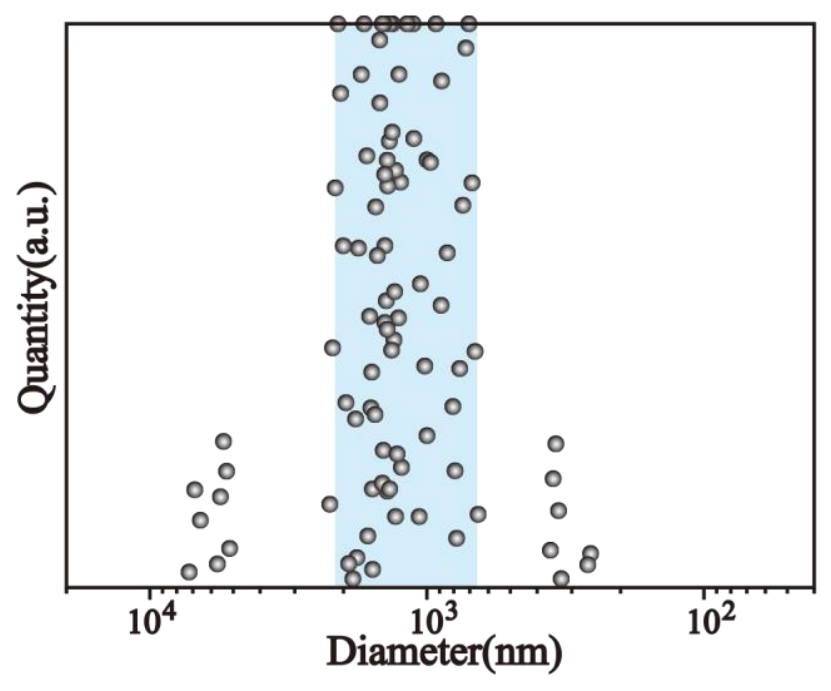

Figure S1. Particle size distribution of GO nanosheets.
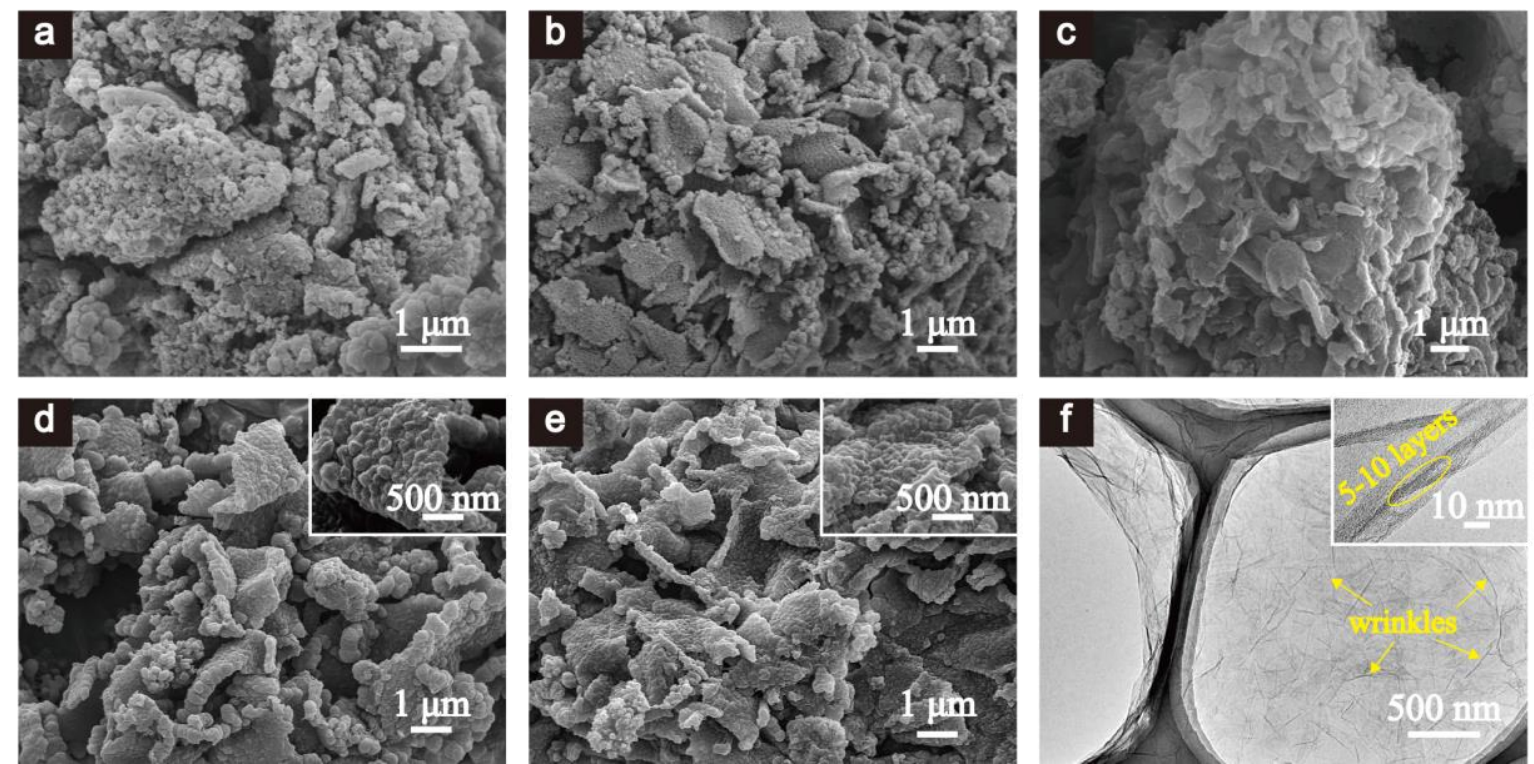

Figure S2. SEM images of P/G-0.1 (a), P/G-0.5 (b), P/G-1 (c), and PAN/GO nanocomposites treated with liquid nitrogen (d) and ultrasonic vibration (e). (f) TEM images of GO. 

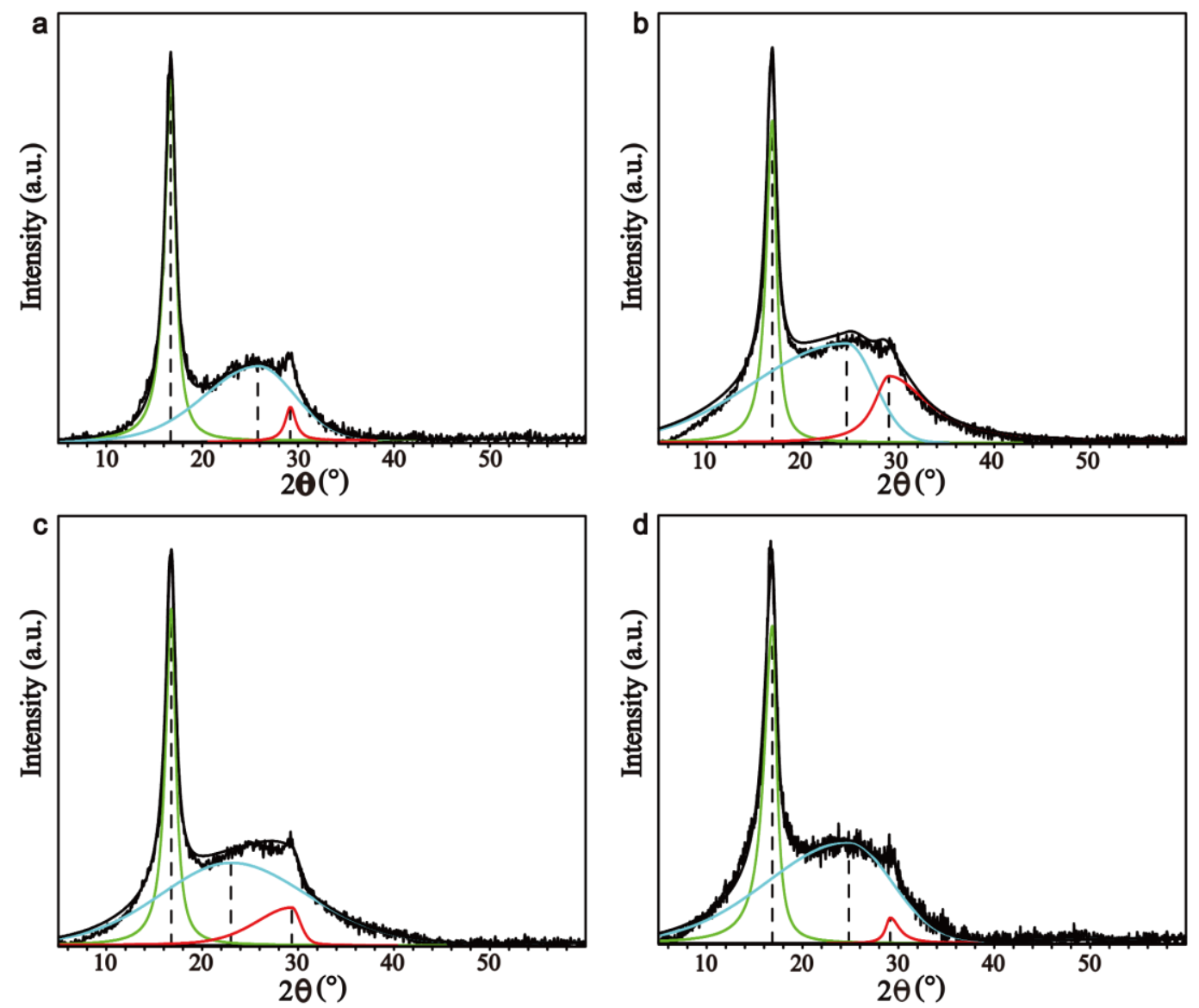

Figure S3. Fitted XRD spectra of polymer products with different GO weight fractions: PAN (a), P/G-0.1 (b), P/G-0.5 (c), and P/G-1 (d). 

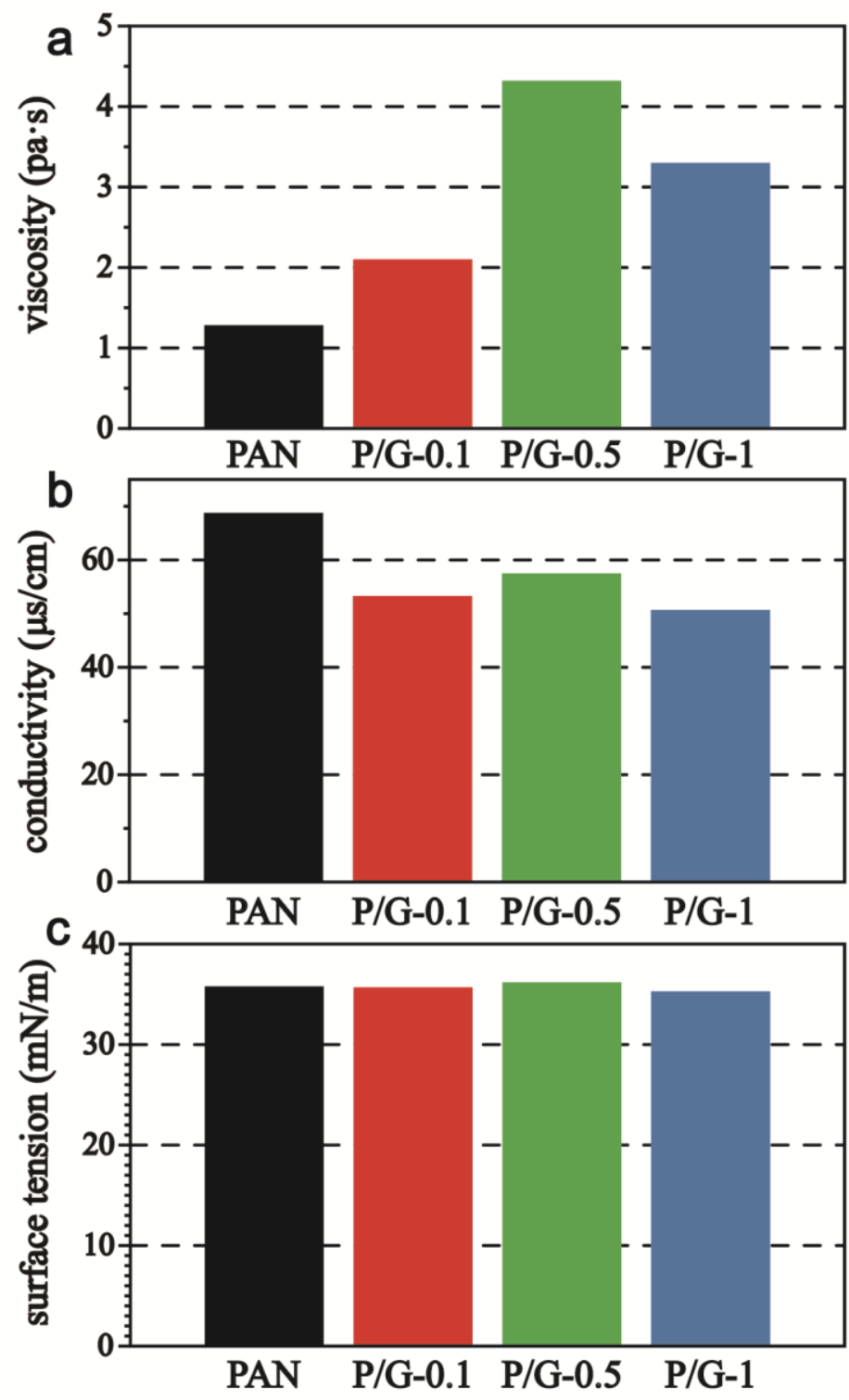

Figure S4. Viscosity (a), conductivity (b) and surface tension (c) of spinning solutions made from polymer products with different GO weight fractions. 

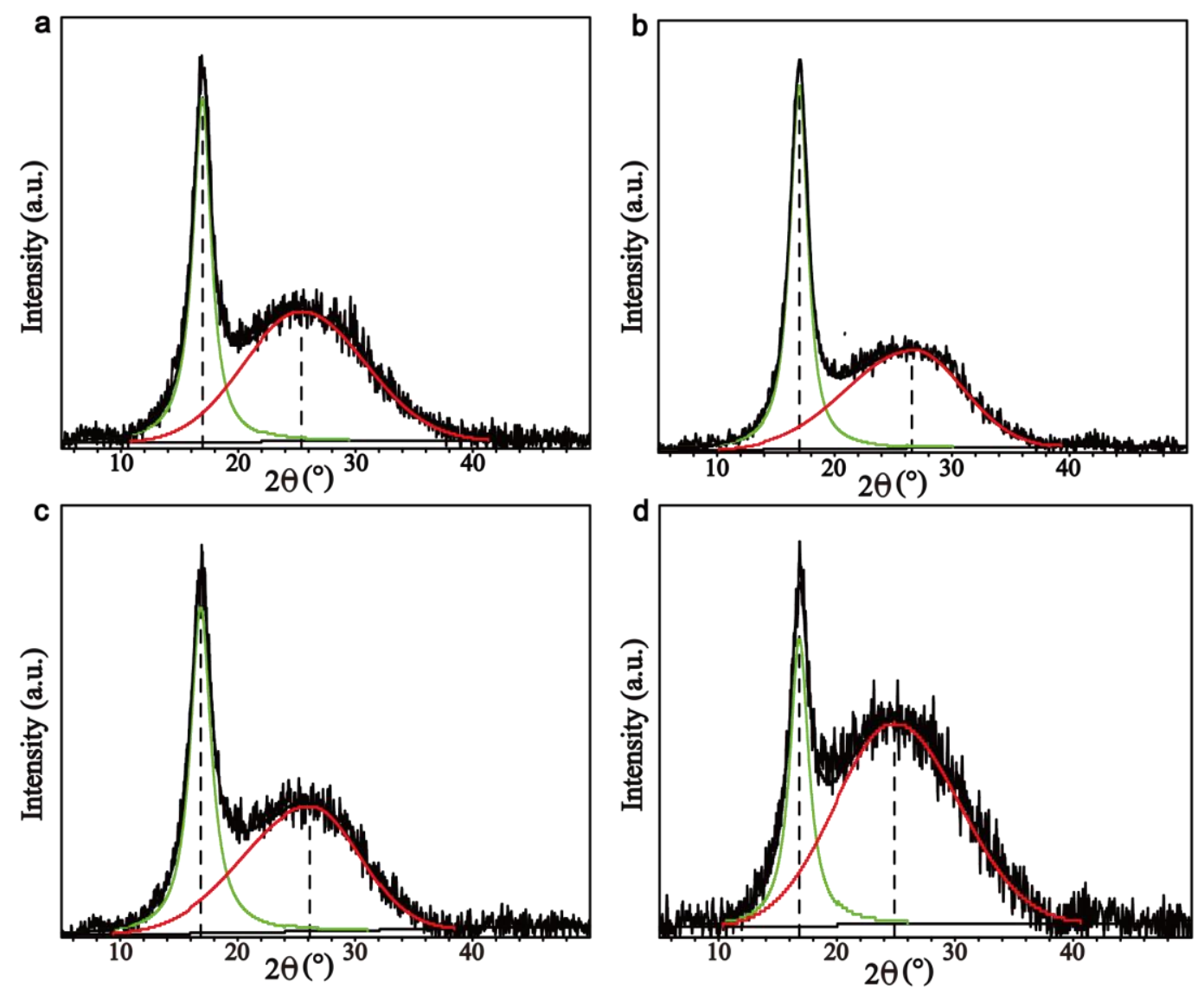

Figure S5. Fitted XRD spectra of the as-spun nanofibers with different GO weight fractions: PAN (a), P/G-0.1 (b), P/G-0.5 (c), and P/G-1 (d).

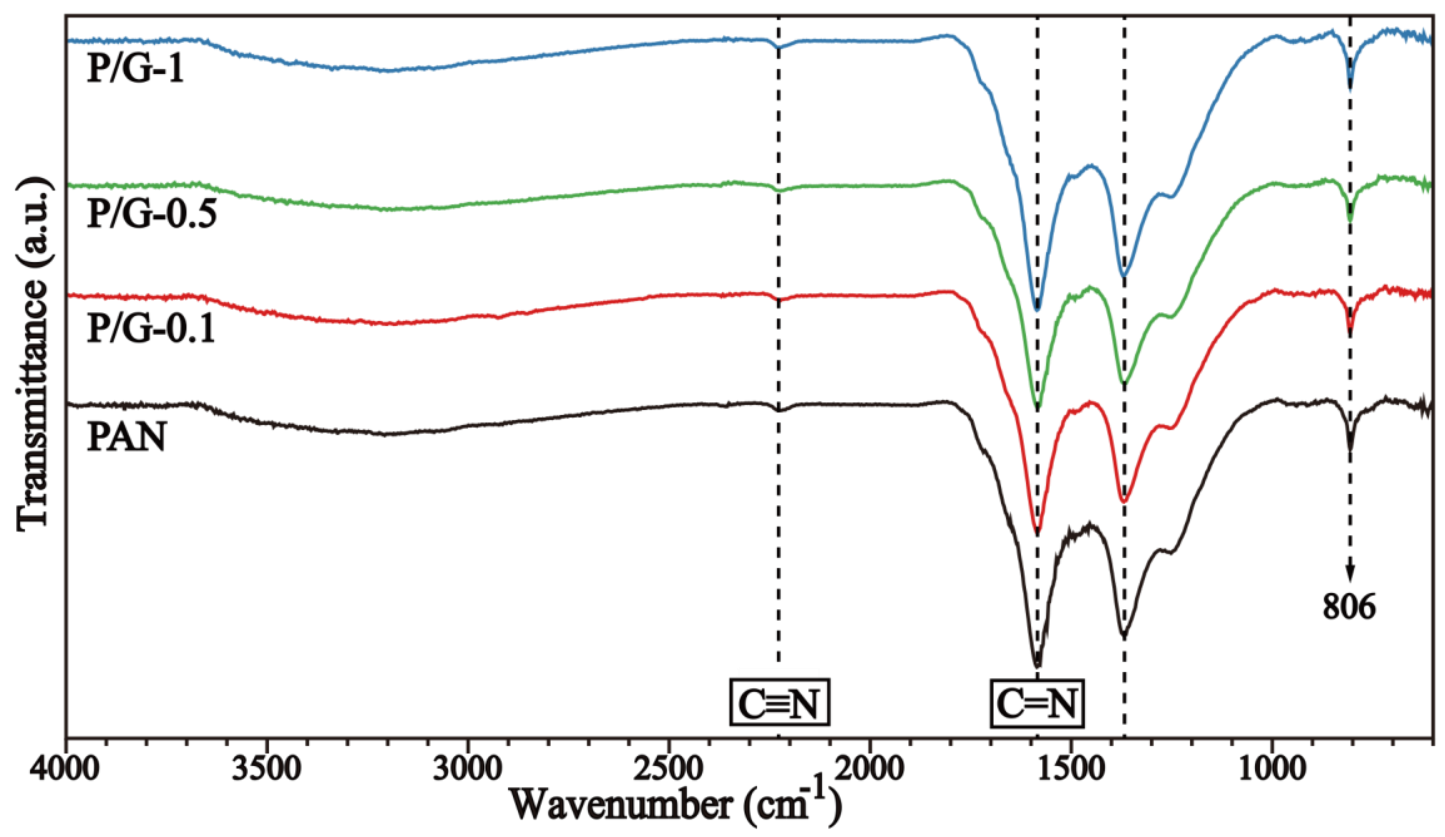

Figure S6. FTIR spectra of stabilized nanofibers with different GO weight fractions. 